\title{
LA JUSTICIA RESTAURATIVA COMO HORIZONTE DE POSIBILIDADES PARA EL PROCESO TRANSICIONAL COLOMBIANO*
}

\author{
Restorative justice as a horizon of possibilities for the Colombian \\ transitional process
}

Julio Gaitán Bohórquez**

Lucia Carcano Fernández ${ }^{* * *}$

Antonio Varón Mejía****

Recepción: 03/01/2019. Aceptación: 05/09/2019

DOI: http://dx.doi.org/10.21017/Rev.Repub.2020.v28.a79

\section{RESUMEN}

Durante los tres más recientes lustros, la justicia restaurativa ha emergido como un nuevo paradigma para abordar el problema del juzgamiento, en particular, de crímenes cometidos en contextos de masivas violaciones a derechos humanos. El centro de la nueva perspectiva son los derechos de las víctimas y la pregunta por mecanismos y prácticas más adecuadas que las tradicionales para recomponer las relaciones sociales quebradas por el conflicto y atender los derechos individuales de maneras alternativas a aquellas que se proponían desde el paradigma retribucionista del derecho penal.

La investigación que da sustento al presente artículo partió de la identificación de dos retos surgidos de las entrevistas con diversos actores, tanto de colectivos de víctimas como con funcionarios de la Jurisdicción Especial de Paz. Por una parte, el reto de la construcción dogmática de la justicia restaurativa y, por otra, el de identificar las claves para su pedagogía y deseable apropiación social.

* El presente artículo se construyó en el marco de una investigación que desarrollaron los investigadores sobre posibles modelos de justicia restaurativa para niños, niñas y adolescentes en el marco de la justicia transicional en Colombia. Enero-septiembre de 2019.

** Julio Gaitán Bohórquez, Ph.D., es Profesor Titular en el área de derecho constitucional en la Universidad del Rosario, de Bogotá, Consultor de Terre des hommes. julio.gaitan@urosario.edu.co@jgaitanb

*** Lucía Carcano Fernández es abogada de la Universidad del Rosario, de Bogotá, consultora de Terre des hommes.

**** Antonio Varón Mejía, Profesor Asistente de la Universidad del Rosario. Consejero de Justicia para Latinoamérica de Terre des hommes. antonio.varon@urosario.edu.co 
De esta manera, emprendimos el ensamblaje de las corrientes teóricas que informan salidas a los conflictos desde la aproximación restaurativa, con los problemas más conspicuos derivados del cambio de paradigma punitivo, así como de las posibles alternativas que las víctimas consideran ajustadas a un marco de aceptabilidad razonable y acordes con sus reclamos de justicia.

El artículo, finalmente, enfoca su propuesta en la construcción colectiva de bienes relacionales como una plausible alternativa a considerar en los desarrollos y en la atención de los reclamos de justicia en contextos de transicionalidad.

Palabras clave: Justicia Restaurativa, Bienes Relacionales, Retribucionismo, Justicia Transicional.

\begin{abstract}
During the most recent three decades, restorative justice has emerged as a new paradigm to address the problem of prosecution, in particular, of crimes committed in contexts of massive human rights violations. The center of the new perspective is the rights of victims and the question of more adequate mechanisms and practices than the traditional ones to recompose the social relations broken by the conflict and attend to individual rights in alternative ways to those proposed from the retribution paradigm. of criminal law.
\end{abstract}

The research that supports this article was based on the identification of two challenges arising from interviews with various actors, both from groups of victims and with officials from the Special Peace Jurisdiction. On the one hand, the challenge of the dogmatic construction of restorative justice and, on the other, to identify the keys to its pedagogy and desirable social appropriation.

In this way, we undertook the assembly of the theoretical currents that inform exits to conflicts from the restorative approach, with the most conspicuous problems derived from the punitive paradigm shift, as well as the possible alternatives that the victims consider fit to a framework of acceptability reasonable and consistent with their claims for justice.

Finally, the article focuses its proposal on the collective construction of relational goods as a plausible alternative to consider in the developments and in the attention to justice claims in transitional contexts.

Key Words: Restorative Justice, Relational Assets, Retributionism, Transitional Justice. 


\section{INTRODUCCIÓN Y PLANTEAMIENTO DEL PROBLEMA}

Durante la última década ha comenzado a implementarse la justicia restaurativa en diferentes escenarios punitivos, como consecuencia de lo cual se ha producido, en primer lugar, un descentramiento de la atención del derecho penal a la figura del victimario, para centrarse en las víctimas y, en segundo lugar, la constatación de la insuficiencia de los recursos punitivos convencionales para lidiar con dinámicas de masivas violaciones de derechos. Esta versión de la justicia, la restaurativa, que pretende ser una alternativa a la justicia retribucionista tradicional, ha sido incluida en varios instrumentos internacionales y nacionales ${ }^{1}$. La novedad de esta aproximación a la comprensión de una nueva forma de entender y proporcionar justicia en muchos escenarios conflictivos y, en particular, en el de la justicia transicional, deriva en que escasamente se encuentran esfuerzos de definición que de manera concisa y explícita delimiten en qué consiste este tipo de justicia, razón por la que a diferentes tipos de procedimientos y aproximaciones se les ha atribuido el adjetivo restaurativo sin mayores consideraciones de lo que esto implica.

Existe, por tanto, un gran reto y tarea, por una parte, de construcción de la institucionalidad y de los referentes dogmáticos que proporcionen herramientas de tratamiento jurídico y, por otra, de desplegar esfuerzos de sensibilización y explicación de los fundamentos conceptuales y prácticos, las implicaciones y beneficios de pensar y operar esta nueva concepción de justicia restaurativa como método para proporcionar a las víctimas la satisfacción de los reclamos de justicia en la que otros modelos han tenido precarios resultados.

El proceso de paz entre el gobierno de Colombia y la guerrilla de las Farc, culminado el 24 de noviembre de 2016, incurre en el señalado problema de indefinición de lo que debe entenderse por justicia restaurativa. El Acuerdo Final señala que

Por lo anterior, uno de los paradigmas orientadores del componente de justicia del SIVJRNR ${ }^{2}$ será la aplicación de una justicia restaurativa que preferentemente busca la restauración del daño causado y la reparación de las víctimas afectadas por el conflicto, especialmente para acabar la situación de exclusión social que les haya provocado la victimización. La justicia restaurativa atiende prioritariamente las necesidades y la dignidad de las víctimas y se aplica con un enfoque integral que garantiza la justicia, la verdad y la no repetición de lo ocurrido. (Acuerdo Final, 2016)

1 Por ejemplo, la Ley 906 de 2004, la Ley 1098 de 2006. Naciones Unidas, y ECOSOC (2002) Restorative Justice, Report of the Secretary General. E/CN.15/2002/5/ entre otros.

2 SIVJRNR: Sistema Integral de Verdad, Justicia, reparación y no Repetición. 
$\mathrm{Y}$ es que, en el momento de ejecutar los acuerdos, particularmente en sede judicial, este vacío presenta una serie de problemas al omitir los indicadores de satisfacción y verificación que debe considerar una reparación en clave restaurativa.

Como se verá más adelante, la Corte Constitucional ha intentado solventar este vacío, pero se ha quedado corta al establecer cuáles son los elementos fundamentales de referencia para la implementación de acciones de reparación en clave restaurativa, cómo debe realizarse la ruptura con el paradigma indemnizatorio-tradicional y la reparación a través de la tarifa legal.

La ley de procedimiento de la Jurisdicción Especial para la Paz presenta en varias oportunidades la posibilidad de la aplicación de la justicia restaurativa, específicamente se refiere a la aplicación de sanciones restaurativas (las denominadas sanciones propias), la posibilidad de realización de una audiencia restaurativa y la reparación en clave restaurativa. Sin embargo, la ley no proporciona luces o criterios acerca de cuáles son los indicadores de satisfacción y verificación de una sanción restaurativa o de una reparación restaurativa. Así mismo, no contempla los tres actores o elementos claves de la justicia restaurativa, la comunidad, la víctima y el agresor. Si bien presenta la oportunidad de que las víctimas participen a través de un apoderado y el agresor de una versión completa y veraz de lo sucedido, no es claro el papel que tiene la comunidad afectada dentro del proceso judicial. En esa medida, se presenta la posibilidad de que las sanciones o modos de reparación se decidan unilateralmente por los jueces sin considerar las necesidades de la comunidad y las víctimas.

Esto podría derivar fácilmente en la continuación del paradigma indemnizatorio patrimonial a través de una suerte de tarifa legal preestablecida y que las sanciones "restaurativas" se conviertan en una mera restricción de la libertad, ignorando o desechando la posibilidad de involucrar factores relacionales a la ecuación.

De modo similar, la normativa del Sistema Integral de Verdad, Justicia y Reparación vigente también ignora el hecho de que las víctimas del conflicto pertenecen a diferentes minorías sujetas a una especial protección constitucional, por lo que las disposiciones restaurativas no pueden ser uniformes, sino que deben de contar con un enfoque diferencial para los niños, niñas y adolescentes, los pueblos indígenas, los pueblos Afro, los pueblos Rom, y miembros de minorías en consideración al género.

A continuación, se identificará cuáles son las principales indeterminaciones que hay frente al contenido de la justicia restaurativa en la normativa colombiana y cómo esto afecta la jurisdicción Especial para la Paz. Posteriormente se 
expondrá la teoría de bienes relacionales como una alternativa para llenar dicho vacío normativo.

\section{INDETERMINACIÓN DE LOS CONTENIDOS DE LA JUSTICIA RESTAURATIVA}

\section{a. Ausencia de definición legal \\ i. Análisis legal}

El ordenamiento jurídico colombiano contempla en diferentes disposiciones la justicia restaurativa, sin embargo, se mantiene un vacío jurídico respecto a los elementos de esta. Para empezar, la Constitución política menciona la Justicia Restaurativa en su artículo 250 al establecer dentro de las funciones de la Fiscalía General de la Nación velar por la protección de las víctimas. Así se limita a establecer que "la ley fijará los términos en que podrán intervenir las víctimas en el proceso penal y los mecanismos de justicia restaurativa" (Constitución Política, artículo 250.7, 1991). Posteriormente, el Código de procedimiento penal (Ley 906 de 2004) estableció en su artículo 518 una definición sumaria de aquello en lo que consiste la justicia restaurativa, de esta manera entiende que:

Se entenderá por programa de justicia restaurativa todo proceso en el que la víctima y el imputado, acusado o sentenciado participan conjuntamente de forma activa en la resolución de cuestiones derivadas del delito en busca de un resultado restaurativo, con o sin la participación de un facilitador. Se entiende por resultado restaurativo, el acuerdo encaminado a atender las necesidades y responsabilidades individuales y colectivas de las partes y a lograr la reintegración de la víctima y del infractor en la comunidad en busca de la reparación, la restitución y el servicio a la comunidad. (Ley 906, 2004. Artículo 518)

A partir de esta definición, el código de procedimiento penal establece que los mecanismos de justicia restaurativa son la mediación, la conciliación prejudicial y el incidente de reparación integral (Ley 906, 2004. Artículo 521). En esa medida, el artículo 521 limita el campo de acción de los procesos restaurativos a escenarios judiciales, despojando la justicia restaurativa de su carácter extraprocesal.

Por su parte, el Código de infancia y adolescencia (Ley 1098 de 2006) menciona en diferentes artículos (artículos 140, 178 y 187) la justicia restaurativa, pero no explica en qué consiste o qué consecuencias tiene que las sanciones tengan una 
finalidad restaurativa. Posteriormente, los Acuerdos de Paz del 2016 retoman el tema de la Justicia Restaurativa al incorporarla como uno de los elementos esenciales en su desarrollo. De esta manera, la ley 1922 de 2018, que establece las reglas de procedimiento para la JEP, menciona en cinco momentos diferentes la justicia restaurativa y el componente restaurativo de las sanciones sin entrar a definir en qué consisten estos componentes.

\section{ii. Análisis jurisprudencial}

La Corte Constitucional ha intentado en diferentes momentos clarificar qué es la justicia restaurativa. El momento más reciente y significativo tuvo lugar con la expedición de la Sentencia C-080 de 2018, que estudia la constitucionalidad de la ley de procedimiento de la JEP. En esta sentencia, la Corte estudia la justicia restaurativa en el ámbito de la justicia transicional. La Corte plantea la justicia restaurativa como una alternativa a la justicia retribucionista, en la que prima la reparación de la víctima y la reconstrucción del tejido social. En esta medida encuentra la existencia de tres enfoques de justicia restaurativa. El primer enfoque está relacionado con la noción de reparación como sinónimo de restitución o indemnización, siempre monetaria. El segundo enfoque gira en torno a los valores de reconciliación entre las partes, aquí se incluyen los programas de mediación entre la víctima y el victimario. El tercer mecanismo de justicia restaurativa son los círculos de sanación y de sentencia. La Corte los asemeja con las Gaccaca en Ruanda y los define como espacios de construcción de la comunidad, orientados a la generación de conciencia y conocimiento colectivo acerca de lo que pasó y que no podrá volver a ocurrir, mediante un proceso de sanación a los victimarios y de reparación para las víctimas.

Luego de este análisis, la Corte concluye que la Justicia Restaurativa es

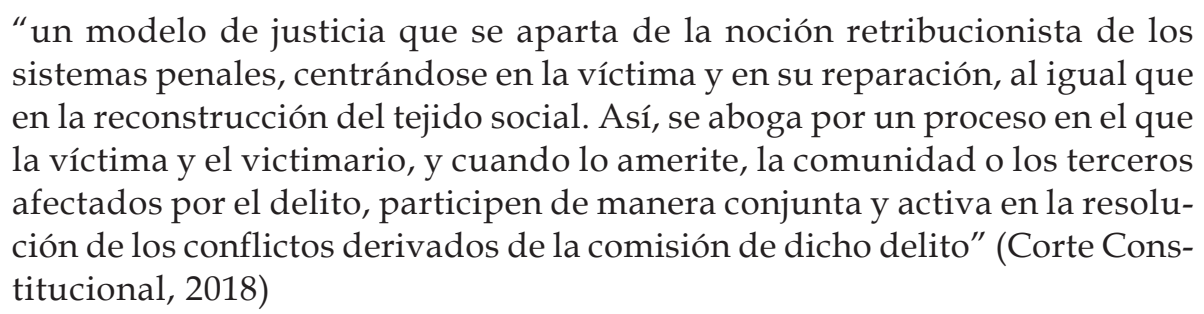

De la definición expuesta por la Corte debe resaltarse el intento por separar la justicia restaurativa del modelo retribucionista procesal hegemónico. Al mismo tiempo es importante el énfasis que se da en el componente de reconstrucción del tejido y en la participación de los diferentes actores afectados por la comisión del delito. Sin embargo, al igual que en la definición del 
Código de procedimiento penal, la definición esbozada no es congruente con el procedimiento general estructurado a lo largo del texto de la ley. Específicamente, contraría el hecho de que el proceso sigue teniendo componentes adversariales en línea con los cuales la participación de las diferentes partes deriva de su acreditación de un ius standi específico, y el hecho de que, en últimas, es un tribunal el encargado de decidir sobre la sanción aplicable al caso.

Similarmente en la sentencia C-017 de 2018 establece cómo

"La justicia restaurativa mira hacia las víctimas, hacia su dignidad, sus específicos daños, necesidades, su general condición histórica de discriminación y los particulares efectos que la violencia les ocasionó. Implica que los mecanismos estatales de la transición ya no están instituidos solo en función de los demás actores del conflicto y la sociedad en general, sino en los derechos y requerimientos de las víctimas. La justicia restaurativa adoptada por el Constituyente, así, está diseñada como una reivindicación de las víctimas y adquiere sentido en virtud de ellas y para ellas".

Precisamente por esto es por lo que la Corte Constitucional resalta en esta providencia que la justicia restaurativa tiene un carácter prioritario sobre las medidas retribucionistas.

Durante el 2018 la Corte Constitucional tuvo otra oportunidad para definir la justicia restaurativa, sin embargo, se limitó a especificar cómo esta se enfoca en "la reconstrucción del tejido social para el goce efectivo de los derechos".

Por otro lado, al analizar la constitucionalidad del Marco Jurídico para la Paz, la Corte se limitó a decir que esta "contempla numerosas y diversas formas: reparaciones, daños remedios, indemnizaciones, restituciones, compensaciones, rehabilitaciones o tributos" manteniéndose en una línea conservadora de lo que es una justicia reparadora, sin adentrar en lo que configura la justicia restaurativa. La confusión se repite en la sentencia C-979 de 2005, que se limita a equiparar la justicia restaurativa con métodos alternativos de solución de conflictos, en esa medida la Corte plantea:

“(i) La justicia restaurativa como mecanismo alternativo para la resolución de conflictos; (ii) las directrices sobre justicia restaurativa, desarrolladas en el seno de la comunidad internacional; (iii) la configuración de la justicia restaurativa en la Ley 906 de 2004; (iv) la mediación como instrumento de aplicación de la justicia restaurativa, (v) la fijación del alcance de los artículos demandados, (vi) la determinación del ámbito de reglamentación que la Constitución confiere al Fiscal General de la Nación". 


\section{iii. Falta de consenso doctrinal}

Por un lado, Sherman \& Strang (2007) establece que la justicia restaurativa es una forma de pensar que involucra lo que es mejor para solucionar las distintas conexiones que hay entre las víctimas del delito, el victimario y el proceso penal. De esta definición deriva tres elementos: (i) la víctima debe estar en el centro; (ii) víctimas y agresores no son enemigos naturales; (iii) víctimas no necesariamente tienen tendencias retributivas; (iv) la cárcel no es necesariamente el castigo elegido por las víctimas.

Para los referidos autores, lo que debe buscarse con la justicia restaurativa es evitar la reincidencia buscando que el agresor sienta empatía con la víctima y viceversa para, de esta forma, reestructurar el tejido social.

Sin embargo, los mismos Sherman \& Strang reconocen que su definición no es absoluta y que existen por lo menos cinco tipos diferentes de justicia restaurativa. (i) Por un lado, está la justicia restaurativa consensual, un proceso en el que todos los actores involucrados en una situación solucionan colectivamente cómo manejar el delito y sus implicaciones para el futuro. Por otro lado, (ii) está la justicia restaurativa como una técnica de resolución de conflictos. Al mismo tiempo (iii) están las aproximaciones que utilizan la justicia restaurativa como una forma de evitar reincidencia vs. las aproximaciones que usan la justicia restaurativa como una herramienta para las situaciones que desbordan la capacidad judicial $\mathrm{y}$, (iv)las aproximaciones que consideran que la justicia restaurativa es exclusiva para víctimas individuales vs. las que consideran que es aplicable también a las situaciones con víctimas colectivas. Por último, (v) están las situaciones que establecen que el confrontar víctima y victimario es un elemento esencial de la justicia restaurativa vs. las vertientes que consideran que la justicia restaurativa puede aplicarse en otros métodos (Sherman \& Strang, 2007).

Por su parte, Gade (2012) presenta una visión similar a la de Sherman \& Strang en la medida en que reconoce la falta de una definición integral de justicia restaurativa, por lo que opta por presentar las ideas centrales de la Justicia Restaurativa. Específicamente, Gade resalta que hay cuatro características comunes en los diferentes movimientos: (i) la idea de que el agresor debe restituir a la víctima, (ii) los procesos penales deben involucrar activamente a todas las partes afectadas, (iii) nociones retributivas están en crisis y, (iv) la justicia restaurativa como justicia dominante en la mayoría del tiempo de la humanidad (Gade, 2012). Al finalizar su exposición, el autor presenta su definición de justicia Restaurativa, a saber:

[Es] Un proceso que busca redefinir el crimen de un quebrantamiento de la ley a un daño generado a otra persona (...) basado en nociones de reparación 
que incentiva a que el agresor, víctima y la comunidad estén involucrados en la resolución de conflicto. (traducido por los autores)

Por su parte, Zehr \& Gohar (2002) optan por limitar la definición de justicia restaurativa a partir de lo que no es esta. En este sentido establecen que: (i) no es sobre perdón o reconciliación, (ii) no es sobre mediación, (iii) no es una garantía para la no reincidencia, (iv) no está diseñado para delitos de menor cuantía (misdemeanor) y, (v) no es el opuesto a retribución (Zehr \& Gohar, 2002).

\section{POSIBLES CAUSAS DE LA INDETERMINACIÓN DE LA JUS- TICIA RESTAURATIVA}

A continuación, se expondrán algunas de las principales causas que han impedido la formación de una definición integral de la justicia restaurativa. Específicamente, haremos énfasis en la fuerza que tiene el populismo punitivo en ordenamientos como el colombiano y la persistencia del paradigma retribucionista en el ordenamiento internacional.

\section{i. Populismo punitivo}

El componente de venganza, de modo explícito o de manera inconfesada, ha estado y sigue estando presente en muchas de las formas punitivas que intentan hacer cuentas con las transgresiones a las reglas que buscan proteger los más valiosos bienes que cada sociedad resguarda. Por tal razón resulta tan contra-intuitivo en nuestra cultura jurídica dominante la posibilidad, o si quiera la idea, de la aplicación de fórmulas no vindicativas de tramitar los resultados de los conflictos, particularmente cuando estos afecten de manera significativa el singular orden establecido en cada comunidad.

No obstante el discurso de humanización de las penas y de resocialización de los transgresores que con altibajos ha tenido tanto suceso durante los últimos doscientos años, las políticas criminales y las prácticas legislativas han vendido y popularizado la falsa idea de que los mecanismos punitivos tradicionales, en los que prevalece la retribución, son los medios más idóneos para proporcionar justicia efectiva a las víctimas, en cuya atención, por cierto, apenas se viene pensando hace un par de décadas. La inercia de la aplicación de mecanismos punitivos retributivos, en particular del encarcelamiento como vía para el restablecimiento del equilibrio entre transgresores y ofendidos, configura un sentido común muy extendido al que es difícil ofrecer alternativas.

El final de la guerra en Colombia entre el Estado y el grupo guerrillero más antiguo del planeta coincidió trágicamente con lo que Wacquant (2009) ha lla- 
mado "una de las mayores transformaciones políticas de los últimos cincuenta años", cual es la irrupción del Estado penal que ha impactado el crecimiento espectacular de la población privada de la libertad.

En este contexto, nada más efectista y disponible para ser creído, que las propuestas de aplicar todo el rigor de la ley penal y los mecanismos de justicia retributiva a los actores de una guerra que llegó a niveles de degradación insoportables. Cualquier alternativa fácilmente es asimilada con impunidad, por lo que las expectativas y aspiraciones de obtención de justicia por esta vía normalmente encuentran grandes audiencias para las propuestas de soluciones punitivas gravosas. Un ejemplo de esto son los discursos utilizados por la oposición al Acuerdo de Paz que considera que una rebaja de penas es sinónimo de impunidad, como olvidar los múltiples discursos del Senador Álvaro Uribe Vélez sobre los indultos que le estaban garantizando a las FARC a través de la $\mathrm{JEP}^{3}$.

Sin embargo, en un contexto como el colombiano, de violaciones masivas de los derechos humanos durante una etapa inusualmente prolongada por décadas, la respuesta del aparato estatal mediante los medios convencionales de persecución y sanción de las violaciones masivas a los derechos humanos es altísimamente improbable, por lo que, sin duda, la prolongación de la guerra era la vía más segura para garantizar la impunidad en uno de los niveles más altos a nivel global. ${ }^{4}$

En un estado de conflicto con niveles cualitativos de degradación insoportables y cuantitativamente difíciles de calcular, las promesas de aplicación de justicia retributiva mediante los mecanismos tradicionales ordinarios no es nada diferente a una forma de instrumentalización del dolor de las víctimas y de prometer resultados irrealizables en contextos de violaciones masivas de derechos humanos, por lo que dichas promesas constituyen manifestaciones de populismo punitivo que no contribuyen en la búsqueda de salidas posibles.

\section{ii. Hegemonía del paradigma punitivo en los estándares internacionales}

La justicia restaurativa ha tenido poco impacto en el funcionamiento de los tribunales penales internacionales en los que ha primado el paradigma

3 Por ejemplo, en agosto 29 de 2019, el ex presidente mencionó a través de un tweet que "Aquí no hubo paz sino el indulto para responsables de delitos atroces"

4 https://www.udlap.mx/cesij/files/IGI-2017.pdf, https://www.amnesty.org/es/ countries/americas/colombia/report-colombia/, https:// noticias.canalrcn.com/amp/ nacional-justicia/impunidad-colombia-del-99-nestor-humberto-martinez/ (Última consulta: 29/09/2019) 
retribucionista (Olasolo, 2017). En esa medida tanto el Tribunal Militar de Nuremberg, los tribunales derivados del Consejo de Control Aliado, el Tribunal Militar Internacional para el Lejano Oriente, los tribunales Ad Hoc para la ex Yugoslavia y Ruanda y la Corte Especial para Sierra Leona presentan un modelo de justicia tradicional en que el papel de las víctimas se encuentra relegado y prima la determinación de la sanción punitiva.

Las Salas Extraordinarias de Camboya comienzan a cambiar este paradigma al permitir la participación de las víctimas como un tipo de parte civil en el proceso penal, en esta medida pueden ser representados por un abogado y participar a través de este en las diferentes etapas procesales. Similarmente, el Estatuto de Roma para la Corte Penal Internacional (ECPI) presenta un modelo atípico de juicio en el que las víctimas pueden participar como partes dentro de las distintas partes del procedimiento (art. 68 del ECPI) y solicitar reparaciones al finalizar el juicio. Esto ha derivado a que en el 2012 el ex presidente de la CPI, Sang-Hyun Song estableciera en un discurso que la CPI integraba el modelo restaurativo y retributivo de justicia (Corte Penal internacional, 2012). Sin embargo, pareciera que el término restaurativo se utilizará ampliamente, al categorizar lo juicios de la CPI de esta forma por el mero hecho de que una de las partes procesales es una representación legal de víctimas (Garbett, 2017). Sin embargo, el modelo procesal no es realmente restaurativo, toda vez que no hay un espacio de comunicación real entre las víctimas y los victimarios, similarmente las víctimas como tal no cuentan con ius standi y deben de contratar un abogado que represente sus intereses en el procedimiento (Garbett, 2017). Adicionalmente, se debe resaltar cómo, de conformidad con el artículo 77, las únicas penas aplicables son penas privativas de la libertad de manera y multas pecuniarias.

\section{A MANERA DE CONCLUSIÓN}

\section{Enfoque relacional de la reparación, elemento central de la justicia restaurativa en contexto de graves violaciones a DDHH}

Pierpaolo Donati y Margaret Archer (2015) definen los bienes relacionales como "aquellas entidades inmateriales (bienes intangibles) que consisten en relaciones sociales que surgen de la reflexividad de los sujetos y que están orientadas a producir y disfrutar juntos, de manera compartida, un bien que de otra manera no podrían obtener". Por su parte, Nusbaum (2001) presenta a estos bienes como aquellas experiencias humanas que son el bien en sí mismo. Estos bienes incluyen diferentes aspectos intangibles de las relaciones de las personas en sociedad y en esa medida se podría deducir que son los más afectados a la hora de un evento violento traumático como una guerra civil que confron- 
ta con grandes magnitudes de violencia a diferentes miembros de la misma comunidad.

Adicionalmente, estos bienes no tienen un propietario singular ni son parte de un colectivo específico, sino que pertenecen a la sociedad de manera genérica (Donati \& Archer, 2015) los cuales son de vital importancia para el mantenimiento estable de una sociedad. Donati \& Archer (2015) incluso llegan a mencionar como la afectación negativa de estos bienes deriva en que "todo el orden social esté empobrecido, mutilado y privado de sangre, con graves daños a las personas y a la organización social en general." (Traducción propia). Al mismo tiempo, estos autores resaltan el hecho de que los bienes relacionales no son bienes fungibles y por tanto no pueden reemplazarse con otros bienes materiales o inmateriales (Donati \& Archer,2015).

En la misma línea de Donati, Martha Nussbaum (1986), Benedetto Gui (1987) y Carole Uhlaner (1989), abordan estos bienes catalogándolos como resultados tangibles de naturaleza comunicativa y afectiva que se producen a través de las interacciones (Gui, 2000). En ese mismos sentido, en tanto que bienes que surgen de las relaciones sociales pueden definirse como productos que del intercambio comunicativo y afectivo de relaciones interpersonales cuyas características esenciales son su no instrumentalidad, reciprocidad y simultaneidad es decir que son bienes que no pueden carecen de un valor económico cuantificable -son bienes de valor intangible- que requieren del intercambio satisfactorio con el "otro" para su nacimiento y se consumen inmediatamente por toda una sociedad o comunidad al momento en que se generan, esto les da un componente de transitoriedad en la medida de existencia de la relación y del intercambio intersubjetivo (Gui, 2000).

Si bien la teoría de la justicia restaurativa todavía no se ha atrevido a mencionar la relación intrínseca que hay entre los objetivos de la justicia restaurativa, la restauración del tejido social afectado por el daño ocasionado, y la existencia de los bienes relacionales, pareciera que en la práctica que lo que se pretende hacer a través de los procesos restaurativos es el restablecimiento de los procesos comunicativos necesarios para el surgimiento de estos bienes intangibles.

En esa medida un proceso real de justicia restaurativa y un proceso de reparación en clave restaurativa, términos ampliamente utilizados en el marco del proceso de paz con las Farc, debería de priorizar la reconstrucción de los bienes relacionales en las regiones más afectadas por el conflicto por encima de simples reparaciones monetarias a través de tarifas legales, que es el modelo predominante hasta ahora. 
Si bien esto podría presentar un reto mayor en cabeza de los encargados de adjudicar las sanciones restaurativas y las reparaciones a las comunidades afectadas, también representa una oportunidad única de reconstrucción real del tejido social. Para esto sería necesario que los jueces tuvieran conocimiento de cuáles son las medidas de satisfacción y verificación que consideran las víctimas y las comunidades afectadas como esenciales para el restablecimiento de los bienes relacionales afectados en el conflicto. Esto en últimas implica una labor más profunda de aproximación entre las diferentes salas de la Jurisdicción Especial para la Paz y las comunidades de las zonas priorizadas de los diferentes casos de la JEP.

De conseguirlo, Colombia sería pionera en la configuración de los marcos necesarios y en proporcionar un abanico de posibilidades y experiencias en el ámbito de la justicia restaurativa desde el Estado en situaciones de transición política, presentando un nuevo camino a otros ordenamientos nacionales y al ordenamiento internacional para romper con el paradigma punitivista que los agobia.

\section{REFERENCIAS}

Acuerdo Final para la Terminación del Conflicto (2016) Disponible en: http:// www.altocomisionadoparalapaz.gov.co/procesos-y-conversaciones/Documentos\% 20compartidos/24-11-2016NuevoAcuerdoFinal.pdf

Claire Garbett (2017) The International Criminal Court and restorative justice: victims, participation and the processes of justice, Restorative Justice, 5:2, 198-220, DOI: $10.1080 / 20504721.2017 .1339953$

Colombia. Corte Constitucional. Sentencia C-017 de 2018.

Colombia. Corte Constitucional. Sentencia C-055 de 2010.

Colombia. Corte Constitucional. Sentencia C-07 de 2018.

Colombia. Corte Constitucional. Sentencia C-080 de 2018.

Colombia. Corte Constitucional. Sentencia C-579 de 2013.

Colombia. Corte Constitucional. Sentencia C-647 de 2001.

Colombia. Corte Constitucional. Sentencia C-979 de 2005.

Donati, P., \& Archer, M. S. (2015). The relational subject. Cambridge University Press. 
Evans, C. (2012). The right to reparation in international law for victims of armed conflict (No. 91). Cambridge University Press.

Gade, C. B. (2013). Restorative justice and the South African truth and reconciliation process. South African Journal of Philosophy, 32(1), 10-35.

GUI, B. y SUGDEN, R. (2005): "Why interpersonal relations matter for economics" en Gui, B. y Sugden, R. (eds.), Economics and social interactions, Accounting for Interpersonal Relations. Cambridge, Cambridge University Press: 1-22.GUI, B. (2000): «Beyond Transactions: On the impersonal dimension of economic reality», Annals of Public and Cooperative Economics vol. 71 (2), 139-169.

GUI, B. (2005): "From transactions to encounters: the joint generation of relational goods and conventional values" en Gui, B. y Sugden, R. (2005): Economics and social interaction, Cambridge, Cambridge University Press.

Nussbaum, M. C. (2001). The fragility of goodness: Luck and ethics in Greek tragedy and philosophy. Cambridge University Press.

Olásolo, H. (2017). Derecho internacional penal, justicia transicional y delitos transnacionales: dilemas políticos y normativos. Tirant lo Blanch.

Sherman, L. W., Strang, H. (2007). Restorative justice: The evidence. Disponible en: https://research.tees.ac.uk/

UHLANER, C. J. (1989): “Relational goods” and participation: Incorporating sociability into a theory of rational action», Public Choice, 62 (3), 253-285.

Wacquant, L. (2009). Castigar a los pobres. El gobierno neoliberal de la inseguridad social. Gedisa.

Zehr, H. (2002). The little book of restorative justice: Revised and updated. Simon and Schuster. 